\title{
BMJ Open Reasons for smoking cessation attempts among Japanese male smokers vary by nicotine dependence level: a cross-sectional study after the 2010 tobacco tax increase
}

\author{
Shinichi Tanihara, Yoshito Momose
}

To cite: Tanihara S, Momose Y. Reasons for smoking cessation attempts among Japanese male smokers vary by nicotine dependence level: a cross-sectional study after the 2010 tobacco tax increase. BMJ Open 2015;5: e006658. doi:10.1136/ bmjopen-2014-006658

- Prepublication history for this paper is available online. To view these files please visit the journal online (http://dx.doi.org/10.1136/ bmjopen-2014-006658).

Received 17 September 2014 Revised 14 February 2015 Accepted 16 February 2015

CrossMark

Department of Preventive Medicine and Public Health, Faculty of Medicine, Fukuoka University, Fukuoka, Japan

Correspondence to Dr Shinichi Tanihara; taniyan@cis.fukuoka-u.ac.jp

\section{ABSTRACT}

Objectives: To examine the association between smoking cessation attempts during the previous 12 months, motivators to quit smoking and nicotine dependence levels among current male smokers after Japan's massive 2010 tobacco tax increase.

Design: Cross-sectional study.

Setting: A self-reported questionnaire about smoking habits, nicotine dependence levels and factors identified as motivators to quit smoking was administered to 9378 employees working at a company located in Fukuoka Prefecture in Japan (as of 1 October 2011).

Participants: A total of 2251 male current smokers 20-69 years old.

\section{Primary and secondary outcome measures:}

Nicotine dependence level assessed by Fagerström Test for Cigarette Dependence (FTCD), smoking cessation attempts during the previous 12 months and motivators for smoking cessation.

Results: The proportion of current smokers who had attempted to quit smoking within the previous 12 months was $40.6 \%$. Nicotine dependence level of current smokers was negatively associated with cessation attempts during the previous 12 months. Motivators for smoking cessation differed by nicotine dependence levels. 'The rise in cigarette prices since October 2010' as a smoking cessation motivator increased significantly at the medium nicotine dependence level (OR 1.44, 95\% Cl 1.09 to 1.90); however, this association was not statistically significant for individuals with high nicotine dependence (OR 1.24, 95\% Cl 0.80 to 1.92). 'Feeling unhealthy' was significantly negatively associated for medium (OR 0.42, 95\% Cl 0.27 to 0.65) and high (OR $0.31,95 \% \mathrm{Cl} 0.14$ to 0.71 ) nicotine dependence levels. Trend associations assessed by assigning ordinal numbers to total FTCD score for those two motivators were statistically significant.

Conclusions: The efficacy of smoking cessation strategies can be improved by considering the target group's nicotine dependence level. For smokers with medium and high nicotine dependence levels, more effective strategies aimed at encouraging smoking cessation, such as policy interventions including increasing tobacco taxes, are needed.

\section{Strengths and limitations of this study}

This study provides information on the relationship between smoking cessation attempts, motivators for quitting smoking and nicotine dependence levels for current smokers after Japan's massive 2010 tobacco tax hike. However, the impact of the tax increase on cessation attempts was not fully investigated because current smokers before the tax increase were not included in the study. No follow-up measures were taken to determine whether the study's respondents actually succeeded in their smoking cessation attempts.

\section{INTRODUCTION}

Smoking is the leading avoidable cause of death worldwide. Many countries take action to reduce smoking-related deaths by educating people about tobacco's harmful effects; ${ }^{1}$ youth tobacco control, including passive smoking control; ${ }^{2}$ disseminating information on tobacco cessation programmes; and increasing tobacco taxes ${ }^{3}$ and prices. ${ }^{4}$ These policies and programmes appear to be effective-overall, the smoking rate is decreasing in developed and developing countries. ${ }^{56}$

In the past two decades, Japan's tobacco tax has increased four times: in December 1998, July 2003, July 2006 and October 2010. The first three increases were relatively low-the price of an ordinary 20-cigarette pack increased by about 20-30 yen (about US\$0.2US\$0.3) at each increase. Given the low taxes in 2008, tobacco was still relatively inexpensive (priced at around 300 yen (US\$2-US\$3) per pack). Thus, smoking prevalence among men remained high in comparison to other Organisation for Economic Co-operation and Development (OECD) countries. 7

However, in October 2010, the price of an ordinary 20-cigarette pack was increased by 
120 yen (US\$1.2), roughly four times the increase of the previous three tax hikes (or an estimated $40 \%$ increase in retail price). According to the National Health and Nutrition Survey in Japan, ${ }^{8}$ the prevalence of regular smoking among men was $38.2 \%, 32.2 \%$ and $32.4 \%$ in 2009, 2010 and 2011, respectively, while that among women was $10.9 \%, 8.4 \%$ and $9.7 \%$, respectively. Thus, these increases in tobacco taxes may have contributed to lower tobacco consumption in Japan, just as was reported in Western European countries. ${ }^{4}$ This may in part be attributable to the tax dissuading young people from smoking. ${ }^{5}$ However, the precise effect of this tax increase on people's smoking cessation attempts in Japan remains unknown.

People quit smoking for numerous reasons other than cigarette price increases, such as health problems associated with smoking and antismoking social pressures. ${ }^{10} 11$ Nevertheless, in order to implement effective strategies aimed at encouraging smoking cessation among Japanese smokers, it is important to determine the varying roles of factors leading to smoking cessation, including smoking cessation attempts, motivating factors for such attempts, and the manner in which nicotine dependence levels affect these attempts. Currently, there is limited availability of information on this topic from current smokers who attempted to quit smoking. A Japanese national survey carried out in 1999 reported that personal health concerns and complications were major motivations for quitting smoking; however, this survey did not provide details on the type of personal health complications/concerns smokers had. ${ }^{12}$ Another survey ${ }^{11}$ reported on the factors related to smoking cessation in former smokers in rural areas of Japan; however, this study did not include current smokers attempting to quit, a population for whom there are little data available. ${ }^{13}$ Additionally, these studies ${ }^{11} 1314$ were conducted before the substantial tobacco tax hike of October 2010, the effect of which has not yet been evaluated. In other words, there are few studies focusing on current smokers after Japan's 2010 tobacco tax hike. Although high nicotine dependence has been shown to be a strong predictor of failure to quit smoking, ${ }^{15}$ the relationship between dependence levels and factors identified as motivators to quit smoking in Japan needs clarification. The present study, therefore, investigates the relationship between nicotine dependence levels and smoking cessation attempts among current smokers, and factors that current smokers identify as motivators to quit smoking following the October 2010 tobacco tax increase.

\section{METHODS AND PROCEDURES}

Study population

A self-reported questionnaire assessing smoking habits, nicotine dependence level and factors identified as motivators to quit smoking was administered to 9378 employees working at a company in Fukuoka Prefecture, Japan (as of 1 October 2011).

\section{Data collection and measurements}

The questionnaire began with queries regarding respondents' age, sex and smoking habits. Individuals who had never smoked were not required to complete the rest of the questionnaire. Former smokers were asked to answer the following additional questions: (1) the brand of cigarettes that they used to smoke, (2) the age at which they ceased smoking (years), (3) their motivators for quitting smoking and (4) any pharmacological therapy used to alleviate nicotine withdrawal. In this study, the definition of former smoker is any person who had once smoked but currently does not. Current smokers were asked (1) to specify the cigarette brand usually smoked, (2) to complete the Fagerström Test for Cigarette Dependence (FTCD), (3) whether they had attempted smoking cessation in the previous 12 months ('No/Yes'), (4) to specify their motivators for quitting smoking and (5) indicate whether they were taking any form of medication for alleviating nicotine withdrawal symptoms (in people attempting to quit).

The FTCD, formerly described as Fagerström Test for Nicotine Dependence (FTND), ${ }^{16}$ a standard questionnaire for assessing physical dependence on nicotine, consists of the following six items: (1) How soon after you wake up do you smoke your first cigarette? ('after $60 \mathrm{~min}$ ', '31-60 min', '6-30 min', 'within 5 min'); (2) Do you find it difficult to refrain from smoking in places where it is forbidden, e.g. in church, at the library, cinema, etc.? ('No/Yes'); (3) Which cigarette would you hate most to give up? ('the first one in the morning', 'all others'); (4) How many cigarettes per day do you smoke? ('10 or less', '11-20', '21-30', '31 or more'); (5) Do you smoke more frequently during the first hours of waking than during the rest of the day? ('No/Yes') and (6) Do you smoke if you are so ill that you are in bed most of the day? ('No/yes'). In scoring the FTCD, the four dichotomous items are scored as 0 or 1 , while the two multiple-choice items are scored from 0 to 3 . The items are then summed to yield a total score of $0-10$. The higher the score, the more dependent the person is on nicotine.

We assessed whether the following nine items were respondents' motivators for quitting smoking with 'yes' or 'no' responses: (1) health problems experienced by relatives or friends, (2) personal health problems, (3) physician recommendation, (4) feeling unhealthy, (5) in the interests of better health, (6) their preferred brand of cigarettes was no longer available for sale, (7) the rise in cigarette prices after October 2010, (8) having a child (or grandchild) and (9) any other motivators.

\section{Statistical analysis}

Of the 9378 candidates, $7899(84.2 \%)$ returned the questionnaire. Of the 7899 participants, we excluded 302 with missing data for sex, age or smoking habits, 76 aged $<19$ because smoking is illegal among individuals under 20 years old in Japan and 19 individuals aged 70 or older because of the small number. We excluded 2830 females because of their low proportion of current 
smokers $(320,11.3 \%)$. Thus, 4672 men aged 20-69 were selected for analysis.

Of the 4672 men aged 20-69 surveyed, 1116 individuals who had never smoked and 1268 former smokers were excluded from the analysis, since FTCD scores were available for current smokers only. Finally, we excluded 33 current smokers with missing data for any of FTCD components and four current smokers with missing data for cessation attempts or motivators for quitting smoking. Thus, the data of 2251 current smokers were analysed in this study.

Responses to the questionnaire were stratified according to respondents' nicotine dependence levels, as defined by the FTCD: low (FTCD score $\leq 3$ ), middle (46 ) and high $(\geq 7)$. First, the proportion of respondents who reported that they had attempted smoking cessation in the previous 12 months (afterward, current smokers with cessation attempts) were expressed as percentages across nicotine dependence levels. Second, the proportion of the motivators for quitting smoking assessed by the nine items among the current smokers with cessation attempts was expressed as percentages across nicotine dependence levels. Third, we selected three motivators for quitting smoking by number of respondents and examined the relationships between nicotine dependence levels and each motivator for quitting smoking. A $\chi^{2}$ test was used to compare the proportion of respondents by the three nicotine dependence levels. Multiple logistic regression analysis estimated the OR with $95 \%$ CIs for the presence of each motivator to quit smoking in the previous 12 months, with low nicotine dependence individuals as the reference. We adjusted for age (10-year categories, 20-29-year-old group as the reference) in the model. Trend associations were assessed by assigning ordinal numbers to a total FTCD score $(0-10)$. A two-tailed $p$ value of less than $5 \%$ was considered statistically significant. All analyses were performed using SPSS V.19 (International Business Machines Corporation, Armonk, New York, USA).

\section{RESULTS}

Of the 2251 current smokers included in our analyses, $913(40.6 \%), 1005(44.6 \%)$ and $333(14.8 \%)$ had low, middle and high FTCD scores, respectively; 914 (40.6\%) reported that they had attempted smoking cessation in the previous 12 months (table 1 ). The 20-29 age group had the lowest proportion of respondents with high nicotine dependence $(7.5 \%)$, while the $50-59$ age group had the highest $(21.4 \%)$. In general, the older groups had more respondents with high nicotine dependence. The $50-59$ age group had the lowest proportion $(33.5 \%)$ of respondents with low nicotine dependence, while the 20-29 age group had the highest $(46.8 \%)$. Overall, the groups with older respondents had the lowest proportions of those with low nicotine dependence.

The proportion of current smokers with cessation attempts among the low, middle and high nicotine dependence groups was $48.0 \%, 37.1 \%$ and $30.9 \%$, respectively, a statistically significant difference. For each age group, the proportion of current smokers with cessation attempts was highest in the low nicotine dependence group. This proportion was lowest in the high nicotine dependence group. This trend was observed across all age groups. For the 30-39, 50-59 and 60-69 age groups, there were significant inverse relations between the proportion of current smokers with cessation attempts and nicotine dependence level. Among the middle and high nicotine dependence groups, the highest proportion of current smokers with cessation attempts was observed in the 20-29 age group (42.5\% and $35.3 \%$, respectively). Furthermore, in the group with low nicotine dependence, the highest proportion of current smokers with cessation attempts was observed in the 60-69 age group (55\%).

Table 2 shows the relationship between motivators and smoking cessation attempts within the previous 12 months by respondents' nicotine dependence levels. About half of current smokers with cessation attempts reported that their motivators were 'for better health' and 'the rise in cigarette prices since October 2010', regardless of their nicotine dependence level. Only $10 \%$ of current smokers with cessation attempts reported that their reasons were for 'personal health problems', 'physician recommendation' and 'feeling unhealthy', regardless of their nicotine dependence level. In the high nicotine dependence group, a higher proportion of current smokers with cessation attempts reported the "health problems of relatives or friends' and 'personal health problems' as two of their primary motivators for quitting smoking. However, the proportion of respondents who cited 'feeling unhealthy' and 'for better health' was highest in the group with low nicotine dependence. Furthermore, in the group with low nicotine dependence, 'the rise in cigarette prices since October 2010' was the least reported reason, while 'for better health' was the most reported. No respondent in the group with high nicotine dependence reported 'having a child (or grandchild)' as their motivator for quitting smoking.

Age-adjusted logistic regression analysis indicating the associations between the three selected motivators for quitting smoking and nicotine dependence levels is shown in table 3. 'Feeling unhealthy' was negatively associated with increases in nicotine dependence levels; the $\mathrm{OR}$ was statistically significant for medium $(\mathrm{OR}=0.42$, $95 \%$ CI $(0.27$ to 0.65$))$ and high ( $\mathrm{OR}=0.31,95 \%$ CI $(0.14$ to 0.71$))$ nicotine dependence levels. 'For better health' was also negatively associated with increases in medium nicotine dependence levels $(\mathrm{OR}=0.71,95 \%$ CI (0.54 to 0.94$))$; however, this association was not statistically significant for individuals with high nicotine dependence (OR=0.88, 95\% CI $(0.57$ to 1.36$))$. 'The rise in cigarette prices since October 2010' was significantly positively associated with an increase in medium nicotine dependence level ( $\mathrm{OR}=1.44,95 \%$ CI (1.09 to 1.90)); however, this association was not statistically 


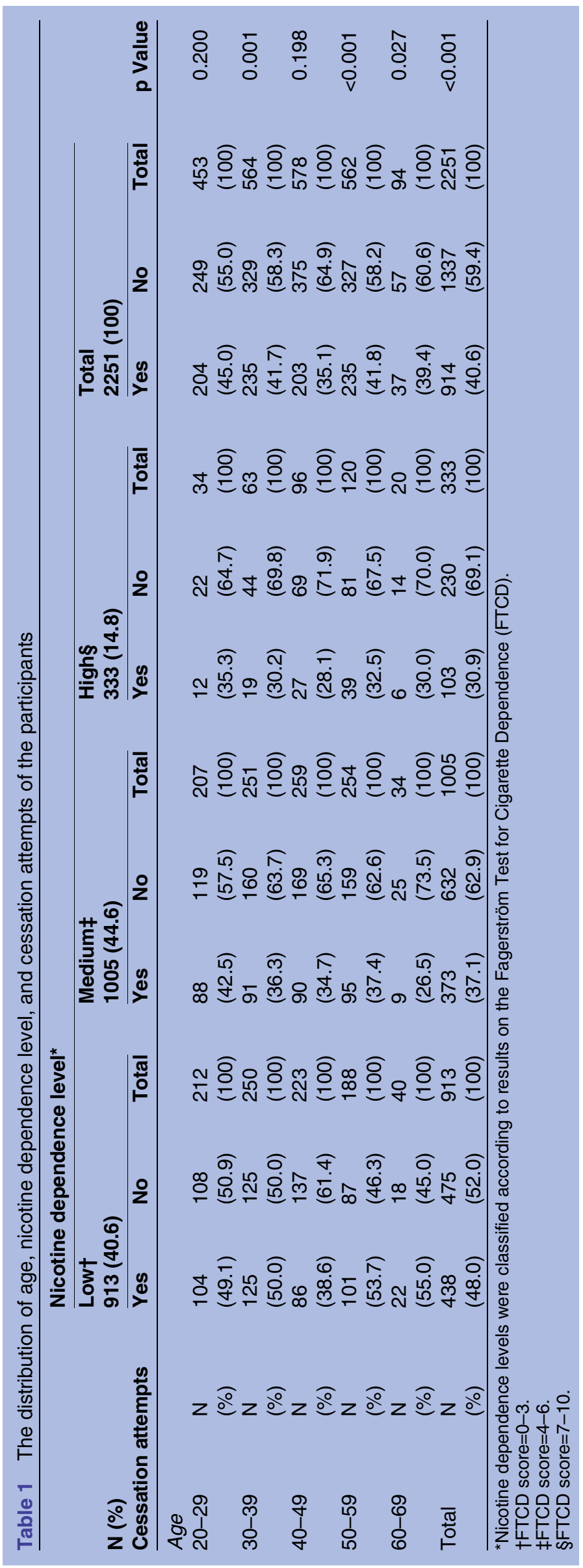

significant for individuals with high nicotine dependence $(\mathrm{OR}=1.24,95 \% \mathrm{CI}(0.80$ to 1.92$))$. Furthermore, trend associations assessed by assigning ordinal numbers to a total score of FTCD (0-10) for all three motivators were statistically significant.

\section{DISCUSSION}

This study investigated the relationships between nicotine dependence level, cessation attempts within the previous 12 months and various motivators for quitting smoking among male current smokers after Japan's 2010 tobacco tax hike. Three major findings emerged. First, $40.6 \%$ of current smokers had attempted smoking cessation within the previous 12 months and nicotine dependence levels were negatively associated with the attempts. Second, respondents' motivators for quitting smoking differed according to their nicotine dependence levels. Third, the proportion of current smokers with cessation attempts who reported 'the rise in cigarette prices since October $2010^{\prime}$ as their motivator for quitting smoking increased with nicotine dependence level.

A national survey in Japan in 1999 showed that $64.7 \%$ of current smokers and $41 \%$ of former smokers acknowledged concerns or problems related to personal health as motivating factors for stopping smoking, respectively. ${ }^{12}$ Furthermore, these motivators were ranked as the first and second leading factors for current and former smokers, respectively. Previous studies ${ }^{10}{ }^{17}$ have also identified personal health concerns, including mild-to-serious personal health problems, and a fear of future illness in the absence of immediate health concerns as predominant motivators for smoking cessation. The present study revealed the relationship between smokers' nicotine dependence levels and motivators for quitting smoking.

In Japan, smoking is still not as restricted in public spaces as it is in some other countries. ${ }^{18}$ In addition, the price of tobacco remains lower than in most developed Western countries. ${ }^{7}$ However, in 2003, the Japanese national government enacted the Health Promotion Law, which requires public facility managers to endeavour to protect non-smokers from the environmental effects of tobacco smoke. Since then, greater restrictions have been placed on smoking in public spaces. In addition, the national government increased the tobacco tax in October 2010. The results of our study suggest that individuals with medium and high nicotine dependence attempted smoking cessation because of the tobacco price. The present findings imply that further legislative changes increasing the cost of cigarettes could be effective in helping people quit smoking.

This study has two major strengths. First, it was conducted after the massive tobacco tax increase in October 2010 , the effects of which could not have been evaluated in previous studies. ${ }^{11} 1314$ The approximately $40 \%$ price increase constituted the highest in Japan within the past two decades. The prevalence of regular smoking among 
Table 2 The proportion of respondents who replied that the motivator is related to the smoking cessation attempts in the previous 12 months

\begin{tabular}{|c|c|c|c|c|c|c|c|c|c|c|}
\hline \multirow[b]{3}{*}{ Motivators to smoking cessation } & \multicolumn{8}{|c|}{ Nicotine dependence level ${ }^{\star}$} & \multirow{3}{*}{$\begin{array}{l}\text { p for trend } \\
\text { (crude) }\end{array}$} & \multirow{3}{*}{$\begin{array}{l}p \text { for } \\
\text { trend (age } \\
\text { adjusted) }\end{array}$} \\
\hline & \multicolumn{2}{|c|}{$\begin{array}{l}\text { Low† } \\
n=438\end{array}$} & \multicolumn{2}{|c|}{$\begin{array}{l}\text { Medium } ¥ \\
n=373\end{array}$} & \multicolumn{2}{|c|}{$\begin{array}{l}\text { High§ } \\
n=103\end{array}$} & \multicolumn{2}{|c|}{$\begin{array}{l}\text { Total } \\
n=914\end{array}$} & & \\
\hline & $\mathbf{n}$ & (\%) & $\mathbf{n}$ & $(\%)$ & $\mathbf{n}$ & (\%) & $\bar{n}$ & (\%) & & \\
\hline Health problems of relatives or friends & 24 & $(5.5)$ & 21 & $(5.6)$ & 8 & $(7.8)$ & 53 & $(5.8)$ & 0.489 & 0.469 \\
\hline Personal health problems & 35 & $(8.0)$ & 33 & $(8.8)$ & 12 & (11.7) & 80 & $(8.8)$ & 0.372 & 0.805 \\
\hline Physician recommendation & 39 & (8.9) & 33 & $(8.8)$ & 12 & $(11.7)$ & 84 & $(9.2)$ & 0.407 & 0.950 \\
\hline Feeling unhealthy & 82 & (18.7) & 33 & $(8.8)$ & 7 & $(6.8)$ & 122 & (13.3) & $<0.001$ & $<0.001$ \\
\hline For better health & 244 & $(55.7)$ & 176 & $(47.2)$ & 55 & $(53.4)$ & 475 & (52.0) & 0.028 & 0.018 \\
\hline Stopped selling my brand of cigarettes & 7 & $(1.6)$ & 9 & $(2.4)$ & 1 & $(1.0)$ & 17 & $(1.9)$ & 0.999 & 0.965 \\
\hline Rise in cigarette prices since October 2010 & 207 & $(47.3)$ & 209 & $(56.0)$ & 51 & $(49.5)$ & 467 & (51.1) & 0.092 & 0.023 \\
\hline Having a child (or grandchild) & 24 & $(5.5)$ & 26 & $(7.0)$ & 0 & $(0.0)$ & 50 & $(5.5)$ & 0.088 & 0.243 \\
\hline Any other motivations & 33 & (7.5) & 33 & (8.8) & 5 & (5.8) & 71 & $(7.9)$ & 0.963 & 0.929 \\
\hline $\begin{array}{l}\text { Age (by } 10 \text {-year age groups) was adjusted by } \mathrm{m} \\
\text { *Nicotine dependence levels were classified acc } \\
\text { †FTCD score }=0-3 \text {. } \\
\text { †FTCD score }=4-6 . \\
\text { §FTCD score }=7-10 .\end{array}$ & le I & tic re & sion & nalysis & & & & (FT & & \\
\hline
\end{tabular}

men and women decreased significantly after the price increase. ${ }^{8}$ A 1-year follow-up survey conducted from 2005 to 2006 in Japan ${ }^{14}$ showed that $23 \%$ of smokers at the time reported that they had attempted to quit smoking at least once in the previous year. In a US study, ${ }^{19} 29 \%$ of respondents reported that the 2009 federal tobacco tax increase helped initiate their attempts to quit smoking. In this study, $40 \%$ of current smokers reported that they had attempted smoking cessation in the previous 12 months. It can be hypothesised that the increase in tobacco tax changed smoking cessation attempts among the Japanese male workers included in the current study sample. Smoking cessation attempts may have been affected by the extent of the tax increase and the resulting rise in cigarette retail prices. ${ }^{3} 469$ Further examination of the influence of tobacco tax increases on smoking cessation in Japan is required.

Second, this study revealed that the reasons for smoking cessation attempts vary by nicotine dependence level. High nicotine dependence strongly predicted failure to quit smoking ${ }^{15}$ or the cost-effectiveness of smoking cessation programmes. ${ }^{20}$ As an employee who feels ready and capable of changing his behaviour has needs and preferences that significantly differ from one who is not at that stage, ${ }^{21}$ workplace smoking cessation interventions that employ only one method ${ }^{22}$ do not generally have positive effects on the smoking cessation attempts of all employees. The current results showed that nicotine dependence is related to motivators for smoking cessation attempts. Thus, the efficacy of any smoking cessation intervention can be improved by considering the target group's level of nicotine dependence.

Four major limitations were identified in this study. First, as this was a cross-sectional study conducted retrospectively, the participants may not recall all attempts for smoking cessation in the previous 12 months. However, we can interpret that their intention to quit is low when the participants do not recall their attempts. Therefore, excluding the quit attempts that the participants could not recall does not affect the interpretation of the study results. This study was conducted in October 2011 and analysed current smokers at that time. Thus, the temporal relationship between the exposure and the outcome should be evaluated cautiously. However, because the subjects of this study were current smokers at the time that this study was conducted, we believe that fluctuation in nicotine dependence is negligible. Thus, temporal relationships between nicotine dependence level and the motivators, and smoking cessation attempts, do not affect the interpretation of the study results.

In the USA, longitudinal studies have been conducted on the effects of a $10 \%$ increase in the retail price of a pack of cigarettes (following the 2009 federal tobacco tax increase) $;{ }^{19}$ however, in Japan, prior investigations of the relationship between retail prices of cigarettes and motivators for smoking cessation among current smokers in Japan were conducted before the 2010 tobacco tax increase. ${ }^{11} 1314$ Thus, the effects of a single $40 \%$ increase in retail prices of cigarettes in Japan are still not sufficiently clear. Further, the association between the number of cigarettes smoked per day and smoking cessation has previously been elucidated; ${ }^{13} 141920$ nevertheless, evaluations of the link between reasons behind smoking cessation and precise nicotine dependence are limited. ${ }^{4}$ Although temporal relationships between the tobacco tax increase and smoking cessation attempts must be carefully evaluated, the method employed for measuring nicotine dependence in this study was widely recognised. A longitudinal evaluation that considers the amount of tax increase, nicotine dependence and smoking cessation attempts must be undertaken.

The second limitation is that those smokers who successfully quit smoking before October 2011 were not included in this study because physical dependence on 


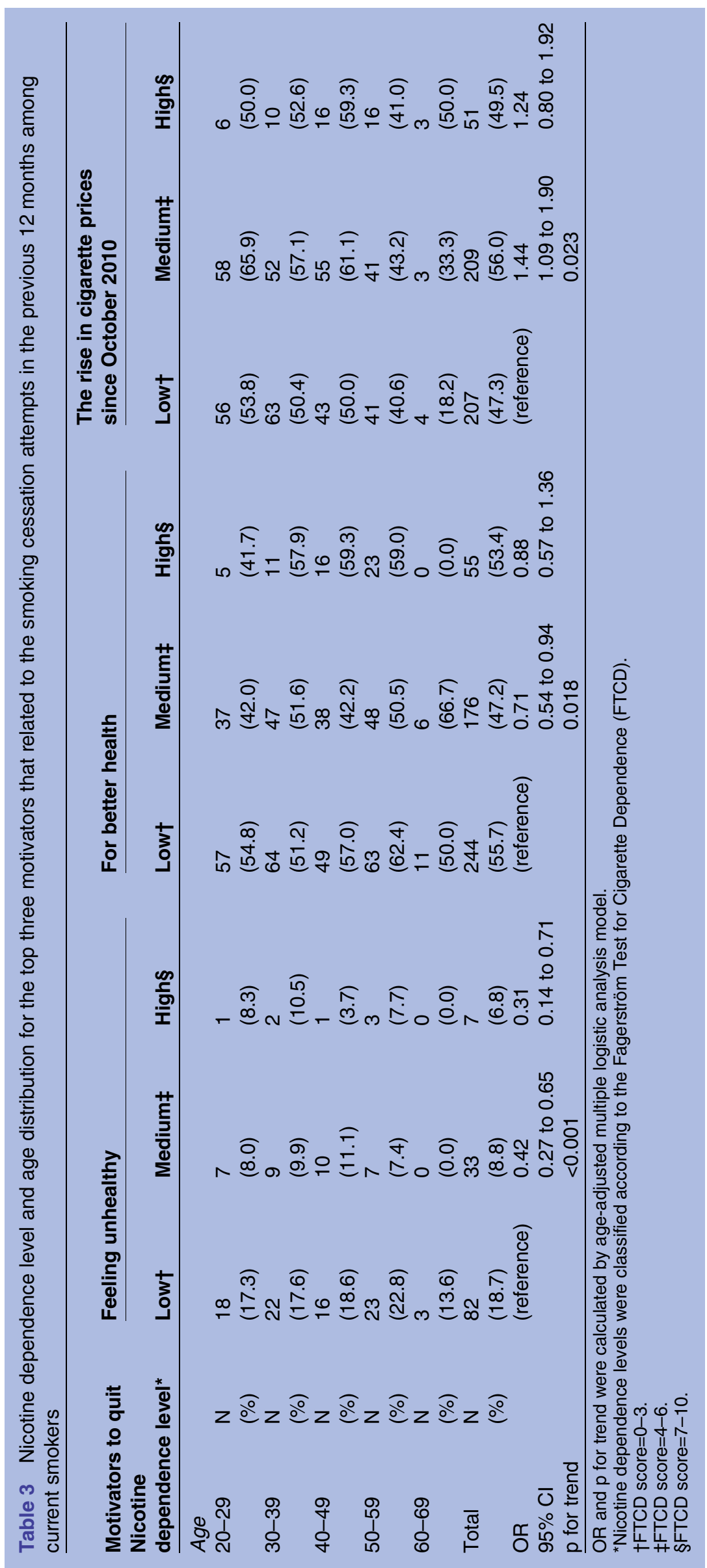


nicotine assessed by the FTCD was available for current smokers only. It is rational to hypothesise that smoking cessation attempts are stronger in smokers who successfully quit smoking than in smokers who continued smoking. Thus, the association between smoking cessation attempts during the previous 12 months and nicotine dependence may be underestimated in this study because the participants in this study were smokers who continued smoking until this study was conducted. This means that the exclusion of the smokers who successfully quit smoking before this study does not influence the interpretation of the results. However, we could not estimate the magnitude of the association between smoking cessation attempts during the previous 12 months and nicotine dependence among the excluded participants. A longitudinal evaluation to investigate the impact of the nicotine dependence level and success in smoking cessation must be undertaken.

The third limitation is that the study sample consisted of only male Japanese workers at a specific company. Therefore, the data analysed were not necessarily representative of the total population. However, we can perhaps assume that the current results have broader applicability to the development and design of workplace smoking cessation interventions, as all respondents in this study were employed on a full-time basis.

Fourth, socioeconomic status was not analysed in this study. It has previously been reported that socioeconomic status, especially income, is related to attitudes towards smoking cessation. ${ }^{19}{ }^{23}$ However, the influence of wage differences on smoking cessation challenges could be disregarded in this study because an age-adjusted analysis (multiple logistic regression) was performed. Income levels are strongly related to respondents' age because of Japan's traditional seniority-based wage system, which had been adopted in the participating company. It has previously been demonstrated that lower education levels, income levels and cigarette consumption are associated with perceptions that the tobacco tax increase is helpful in aiding smoking cessation. ${ }^{19}$

In conclusion, the efficacy of smoking cessation strategies can be improved by considering the target group's nicotine dependence level. A longitudinal evaluation of strategies focused on current smokers with high nicotine dependence levels should be undertaken.

Contributors ST conceived and coordinated the study, participated in its design, collected and interpreted the data, conducted data analysis and drafted the manuscript. YM participated in the study design and data collection, interpreted the data and helped to draft the manuscript. Both the authors approved the final version of the manuscript.

Funding This study was funded by a Grant-in-Aid from the Ministry of Health, Labour and Welfare of Japan (Comprehensive Research on Cardiovascular and Life-style Related Disease: H22-Junkankitou [Seisyuu]-Ippan-012).

Competing interests None.

Ethics approval The research protocol was approved by the Institutional Review Committee of Fukuoka University.

Provenance and peer review Not commissioned; externally peer reviewed.
Data sharing statement No additional data are available.

Open Access This is an Open Access article distributed in accordance with the Creative Commons Attribution Non Commercial (CC BY-NC 4.0) license, which permits others to distribute, remix, adapt, build upon this work noncommercially, and license their derivative works on different terms, provided the original work is properly cited and the use is non-commercial. See: http:// creativecommons.org/licenses/by-nc/4.0/

\section{REFERENCES}

1. Schane RE, Prochaska JJ, Glantz SA. Counseling nondaily smokers about secondhand smoke as a cessation message: a pilot randomized trial. Nicotine Tob Res 2013;15:334-42.

2. Blanch C, Fernandez E, Martinez-Sanchez JM, et al. Impact of a multi-level intervention to prevent secondhand smoke exposure in schoolchildren: a randomized cluster community trial. Prev Med 2013;57:585-90.

3. MacFarlane K, Paynter J, Arroll B, et al. Tax as a motivating factor to make a quit attempt from smoking: a study before and after the April 2010 tax increase. J Prim Health Care 2011;3:283-8.

4. Ross H, Blecher E, Yan L, et al. Do cigarette prices motivate smokers to quit? New evidence from the ITC survey. Addiction 2011;106:609-19.

5. Guindon GE. The impact of tobacco prices on smoking onset in Vietnam: duration analyses of retrospective data. Eur J Health Econ 2014;15:19-39.

6. Salti N, Chaaban J, Nakkash R, et al. The effect of taxation on tobacco consumption and public revenues in Lebanon. Tob Control 2015;24:77-81.

7. Organisation for Economic Co-operation and Development. OECD Health Statistics 2014. http://stats.oecd.org/index.aspx? DataSetCode=HEALTH_STAT (accessed 12 Sept 2014).

8. Ministry of Health, Labour and Welfare. The National Health and Nutrition Survey in Japan. http://www.mhlw.go.jp/stf/houdou/2r985200 0002q1st-att/2r9852000002q1wo.pdf (accessed 12 Sep 2014).

9. Dunlop SM, Perez D, Cotter T. Australian smokers' and recent quitters' responses to the increasing price of cigarettes in the contex of a tobacco tax increase. Addiction 2011;106:1687-95.

10. Aubin HJ, Peiffer G, Stoebner-Delbarre A, et al. The French Observational Cohort of Usual Smokers (FOCUS) cohort: French smokers perceptions and attitudes towards smoking cessation. BMC Public Health 2010;10:100.

11. Nakamura K, Sakurai M, Nishijo M, et al. Characteristics of smoking cessation in former smokers in a rural area of Japan. Int J Prev Med 2012;3:459-65.

12. Ministry of Health, Labour and Welfare. The survey for smoking and health problems in Japan. http://www1.mhlw.go.jp/houdou/1111/ h111211.html\#n05 (accessed 12 Sep 2014).

13. Goto R, Takahashi $Y$, Ida T. Changes in smokers' attitudes toward intended cessation attempts in Japan. Value Health 2011;14:785-91.

14. Hagimoto A, Nakamura M, Morita T, et al. Smoking cessation patterns and predictors of quitting smoking among the Japanese general population: a 1-year follow-up study. Addiction 2010;105:164-73.

15. Japuntich SJ, Leventhal AM, Piper ME, et al. Smoker characteristics and smoking-cessation milestones. Am J Prev Med 2011;40:286-94.

16. Heatherton TF, Kozlowski LT, Frecker RC, et al. The Fagerström Test for Nicotine Dependence: a revision of the Fagerström Tolerance Questionnaire. Br J Addict 1991;86:1119-27.

17. McCaul KD, Hockemeyer JR, Johnson RJ, et al. Motivation to quit using cigarettes: a review. Addict Behav 2006;31:42-56.

18. Yorifuji T, Tanihara S, Takao S, et al. Regional disparities in compliance with tobacco control policy in Japan: an ecological analysis. Tob Control 2011;20:374-9.

19. Choi K, Boyle RG. Minnesota smokers' perceived helpfulness of 2009 federal tobacco tax increase in assisting smoking cessation: a prospective cohort study. BMC Public Health 2013;13:965.

20. Nakamura K, Sakurai M, Miura K, et al. Nicotine dependence and costeffectiveness of individualized support for smoking cessation: evidence from practice at a worksite in Japan. PLoS One 2013;8:e55836.

21. Carroll C, Rick J, Leaviss J, et al. A qualitative evidence synthesis of employees' views of workplace smoking reduction or cessation interventions. BMC Public Health 2013;13:1095.

22. Hishida A, Terazawa T, Mamiya T, et al. Efficacy of genotype notification to Japanese smokers on smoking cessation-an intervention study at workplace. Cancer Epidemiol 2010;34:96-100.

23. Douglas L, Szatkowski L. Socioeconomic variations in access to smoking cessation interventions in UK primary care: insights using the Mosaic classification in a large dataset of primary care records. BMC Public Health 2013;13:546. 